\title{
LETTERS
}

\section{Thrombophilia testing in venous thromboembolism}

Khan and colleagues ${ }^{1}$ advise thrombophilia testing for patients who are going to stop anticoagulation after a deep vein thrombosis or pulmonary embolism. They also advise that patients with strong thrombophilia (antithrombin deficiency, antiphospholipid antibodies or combined thrombophilias) should continue anticoagulation indefinitely.

This advice of Khan and colleagues is not supported by the current evidence.

Dr. Jean Connors, in a recent New England Journal of Medicine article, argued strongly against thrombophilia testing to decide the duration of anticoagulation therapy. ${ }^{2}$

Duration of anticoagulation should be made on the basis of whether venous thromboembolism was provoked or unprovoked. Patients with unprovoked venous thromboembolism should continue anticoagulation for the rest of their lives, irrespective of whether they have thrombophilia or not. Similarly, patients with a provoked venous thromboembolism should be anticoagulated for three months, irrespective of the presence or absence of thrombophilia. These recommendations are based on available data that show no substantial difference in rates of recurrent venous thromboembolism between patients with and without thrombophilia. ${ }^{3}$
Thrombophilia testing is also used inappropriately to decide about primary or secondary thromboprophylaxis. Here, again, there is no evidence that patients with thrombophilia should be treated differently from those without thrombophilia or a family history of recurrent venous thromboembolism. Both groups should receive thromboprophylaxis on the basis of established risk factors for venous thromboembolism (e.g., surgery and type of surgery, active cancer, immobility). Risk stratification tools such as DASH (D-dimer, Age, Sex, Hormonal therapy) ${ }^{4}$ and HERDoo2 (Hyperpigmentation, Edema, or Redness in either leg; D-dimer level $\geq 250 \mu \mathrm{g} / \mathrm{L}$; Obesity with body mass index $\geq 30$; or Older age, $\geq 65 \mathrm{yr})^{5}$ do not incorporate thrombophilia status into any of these tools.

It becomes even more complicated when we know that thrombophilia testing does not identify all patients with increased risk of venous thromboembolism and hence may give a false sense of safety.

As mentioned by Connors, even patients with homozygous factor $V$ Leiden or prothrombin gene mutation, or those who have protein $\mathrm{C}$, protein $\mathrm{S}$ or antithrombin deficiency, do not require lifelong anticoagulation. ${ }^{6}$

Whether venous thromboembolism is provoked or unprovoked determines the duration of anticoagulation, irrespective of thrombophilia status or family history of recurrent venous thromboembolism.

Patients with antiphospholipid antibody syndrome fall into a different category, and antiphospholipid screening may be appropriate in the right clinical context.

\section{Habib U. Rehman MBBS}

Physician, Saskatchewan Health

Authority, Regina, Sask.

Cite as: CMAJ 2018 February 12;190:E171. doi: $10.1503 / \mathrm{cmaj} .733532$

\section{References}

1. Khan F, LeGal G, Rodger MA. Stopping anticoagulation in a woman with unprovoked venous thromboembolism. CMAJ 2017;189: E1112-4.

2. Connors JM. Thrombophilia testing and venous thrombosis. N Engl J Med 2017;377:1177-87.

3. Coppens M, Reijnders JH, Middeldorp S, et al. Testing for inherited thrombophilia does not reduce the recurrence of venous thrombosis. J Thromb Haemost 2008;6:1474-7.

4. Rodger MA, Kahna SR, Wells PS, et al. Identifying unprovoked thromboembolism patients at low risk for recurrence who can discontinue anticoagulant therapy. CMAJ 2001;179:417-26.

5. Tosetto A, Lorio M, Marcucci M, et al. Predicting disease recurrence in patients with previous unprovoked venous thromboembolism: a proposed prediction score (DASH). J Thromb Haemost 2012;10:1019-25.

6. Baglin T, Luddington R, Brown K, et al. Incidence of recurrent venous thromboembolism in relation to clinical and thrombophilic risk factors: prospective cohort study. Lancet 2003;362:523-6.

Competing interests: None declared. 